\title{
Screening for Maternal Postpartum Depression During Infant Hospitalizations
}

\author{
Margaret J. Trost, MD,2*, Kira Molas-Torreblanca, DO ${ }^{1,2}$, Carol Man, BS², Ernesto Casillas, BS², Hoda Sapir, LCSW², \\ Sheree M. Schrager, PhD, MS 1
}

${ }^{1}$ Division of Hospital Medicine, Children's Hospital Los Angeles, Los Angeles, California; 2University of Southern California, Keck School of Medicine, Los Angeles, California; 'Division of Social Work, Children's Hospital Los Angeles, Los Angeles, California.

BACKGROUND: Postpartum depression is common and adversely affects children of afflicted mothers; postpartum depression recognition and treatment may improve outcomes. Hospitalization represents a potential health encounter for expanding screening and intervention.

OBJECTIVE: We aimed to assess for postpartum depression at infant hospitalization and examine postpartum depression risk factors in this population.

DESIGN, SETTING, AND PARTICIPANTS: We conducted a prospective observational study of 310 English- or Spanishspeaking women with an infant aged 2 weeks to 1 year admitted to a pediatric hospitalist service at a large urban freestanding children's hospital.

MEASUREMENTS: Mothers completed demographic questionnaires, a maternal-infant bonding scale, and the Edinburgh Postpartum Depression Scale (EPDS). Mothers with an EPDS score of 10 or higher (positive screen) received counseling and mental health referrals. Postenrollment calls followed trends in EPDS score and resource utilization.
Multivariate logistic regression assessed relationships among risk factors and positive screens.

RESULTS: Eighty-seven mothers (28\%) were EPDS+. Only $14.6 \%$ reported appropriate prior depression screening. Maternal factors associated with EPDS+ were poor social support (4.40, interquartile range $[\mathrm{IQR}]=2.27-8.53$ ) and history of psychiatric diagnoses $(5.02, \mathrm{IQR}=2.49-10.15)$. Having an infant with neurodevelopmental comorbidities was associated with EPDS + screens $(2.78$, IQR $=1.03-$ 7.52). Of 21 initially EPDS + mothers reached by phone, $8(38 \%)$ utilized their doctor or referral resource, resulting in lower EPDS scores $(F(1,19)=5.743, P<0.05)$ compared to those not seeking help.

CONCLUSION: Postpartum depression screening during infant hospitalizations captures women previously unscreened. Low social support, past psychiatric diagnoses, or having infants with neurodevelopmental problems may increase postpartum depression risk. Journal of Hospital Medicine 2016;11:840-846. (C) 2016 Society of Hospital Medicine
Maternal postpartum depression occurs in 5\% to $25 \%$ of all mothers, and up to $40 \%$ to $60 \%$ in highrisk populations such as low-income women. ${ }^{1-4}$ Children of affected mothers suffer negative health consequences such as decreased physical growth, poor maternal-child bond, problem behavior, and child abuse. ${ }^{5-7}$ Timely recognition of symptoms and treatment may improve child outcomes. ${ }^{8}$ Published guidelines recommend pediatricians screen for postpartum depression at infant 1-, 2-, 4-, and 6-month outpatient visits. ${ }^{9}$ There are no current guidelines for or studies of screening in general inpatient settings, although emergency rooms ${ }^{10}$ and neonatal intensive care units (NICUs) $^{11}$ have been examined. Pediatric

*Address for correspondence and reprint requests: Margaret J. Trost, MD, Division of Hospital Medicine, 4650 Sunset Blvd. \#94, Los Angeles, CA 90027; Telephone: 323-361-7112; Fax: 323-361-8106; E-mail: mtrost@chla.usc.edu

Additional Supporting Information may be found in the online version of this article.

Received: March 17, 2016; Revised: June 17, 2016; Accepted: June 23, 2016

2016 Society of Hospital Medicine DOI 10.1002/jhm.2646

Published online in Wiley Online Library (Wileyonlinelibrary.com). hospitalization may offer an additional opportunity for expanding screening and intervention.

Augmenting outpatient screening practices with additional inpatient screening would have several benefits. Infant health problems have been associated with postpartum depression, and therefore mothers in the hospital may be at higher risk. ${ }^{12}$ Inpatient screening would also improve access to mothers not screened as outpatients. Missed screening could occur due to physician discomfort with screening, time constraints during busy office visits, or noncompliance with recommended visit schedules. ${ }^{13-16}$ Finally, inpatient providers would benefit from understanding the psychosocial milieu of children now under their care. Recent studies note hospital discharges may be improved and readmissions reduced by assessing socioeconomic risk factors during hospitalization. ${ }^{17}$ The evidence-based Peds Effective Discharge: Better Handoff to Home through Safer Transitions Better Outcomes by Optimizing Safe Transitions (PediBOOST) toolkit specifically recommends an assessment of parental psychiatric issues. ${ }^{18}$ Postpartum depression strongly correlates with impaired maternal-child bonding, ${ }^{19}$ which in turn negatively affects mothers' engagement with healthcare providers. ${ }^{20}$ 
This could impact patient education and recommendations provided during hospitalization.

Therefore, we sought to perform postpartum depression screening during infant hospitalizations. Our primary goal was to determine rate of postpartum depression in our population and proportion of women previously unscreened who could be captured by inpatient screening. We additionally aimed to determine the proportion of women with poor maternal-infant bond. Our next goal was to identify maternal or infant factors associated with positive postpartum depression screening. Finally, we performed follow-up calls to determine if in-hospital interventions resulted in formal postpartum depression diagnosis, use of recommended referrals, improved maternal-child bond, and decreased symptoms of depression over time.

\section{METHODS}

\section{Patient Selection}

We conducted a prospective observational study on a convenience sample of mothers at Children's Hospital Los Angeles (CHLA), a large, urban, tertiary care hospital. Biological mothers of infants $<1$ year of age admitted to medical-surgical floors and assigned to pediatric hospitalist teams between April 1, 2013 and July 30, 2014 were eligible for inclusion. Mothers were required to be age 18 years or older and able to speak and read English or Spanish. Mothers of infants aged $<2$ weeks were excluded to avoid confusing postpartum depression with maternal "baby blues," a distinct entity causing milder symptoms of depression that should resolve by 2 weeks. ${ }^{21}$ In an effort to reduce the impact of stress associated with prolonged hospitalization on Edinburgh Postpartum Depression Scale (EPDS) scores, we excluded mothers of children already hospitalized $>72$ hours. Visits from participants who were readmitted or previously enrolled in the study were excluded. All study procedures were approved by the CHLA Institutional Review Board.

\section{Measures}

After giving informed consent, mothers completed demographic forms about themselves and their infants. A 4-item Likert scale assessed self-perceived support from family and friends. Past mental health problems were assessed via 10 -item checklist. Selfreported infant comorbidities and reason for hospitalization were confirmed by chart review for International Classification of Diseases, Ninth Revision diagnoses present on admission and reason for discharge. Next, mothers filled out a maternal-infant bonding scale $(\mathrm{MIB})^{22}$ and the EPDS, ${ }^{23,24}$ which has been validated in both English and Spanish. ${ }^{25}$ There are no formal cutoffs for the MIB; higher scores indicate worse bonding. Out of a possible 30, a score of 10 or higher on the EPDS was considered a "positive" screen, indicating risk for postpartum depression.
Scores less than 10 were "negative" screens, and those mothers were determined not at risk. ${ }^{24}$ The last EPDS question asks, "The thought of harming myself has occurred to me." Any mothers answering "yes, quite often," "sometimes," or "hardly ever" were further interviewed and treated per a suicidality operating protocol.

\section{Counseling and Referral}

All EPDS - mothers were informed of results and did not receive further intervention during hospitalization. For EPDS+ mothers, individual social workers responded to referrals placed by the study team into infant charts and delivered 1-on-1 counseling. Social workers received study education prior to initiation and midway through patient recruitment and provided mothers with an educational handout, referral sheet listing online resources of local mental health clinics accepting postpartum depression patients, and helpline numbers. Mothers who identified a primary doctor were encouraged to follow up with them.

\section{Follow-up}

In order to assess intervention effect over time, all mothers (both EPDS+ and EPDS-) were called 3 and 6 months ( \pm 1 week) postenrollment and rescreened with the EPDS and MIB. They also answered a short survey assessing whether they spoke further to a doctor about postpartum depression; used a referral resource; received a formal postpartum depression diagnosis; and if their children visited the ER, urgent care, or hospital again since discharge. Mothers who again screened EPDS + or newly converted to EPDS+ were provided counseling and referral via phone.

\section{Sample Size Calculation}

A priori power analysis determined a sample size of 310 mothers was required to estimate the rate of postpartum depression at CHLA with 5\% precision and a 95\% confidence level, assuming an estimated prevalence of $27.9 \%$ based on prior studies. ${ }^{26}$ At this prevalence rate, screening 310 mothers was also predicted to yield at least 77 positive screens on the EPDS, yielding an appropriate sample to detect EPDS score improvements over time. This number was based on previous studies showing reduction in EPDS of 35\% following appropriate referral, ${ }^{26,27}$ assuming $15 \%$ attrition at both the 3 month and 6-month follow-up sampling points.

\section{Statistical Analysis}

After data collection was complete, characteristics between EPDS + and EPDS - groups were compared using $\chi^{2}$ tests for dichotomous outcomes and $t$ tests for continuous variables. Multiple logistic regression was then used to compare specific factors associated with positive EPDS screens $(P<0.05)$. Linear regression assessed the relationship between EPDS and MIB scores. Change in average EPDS and MIB scores at the time of first successful follow-up call between 


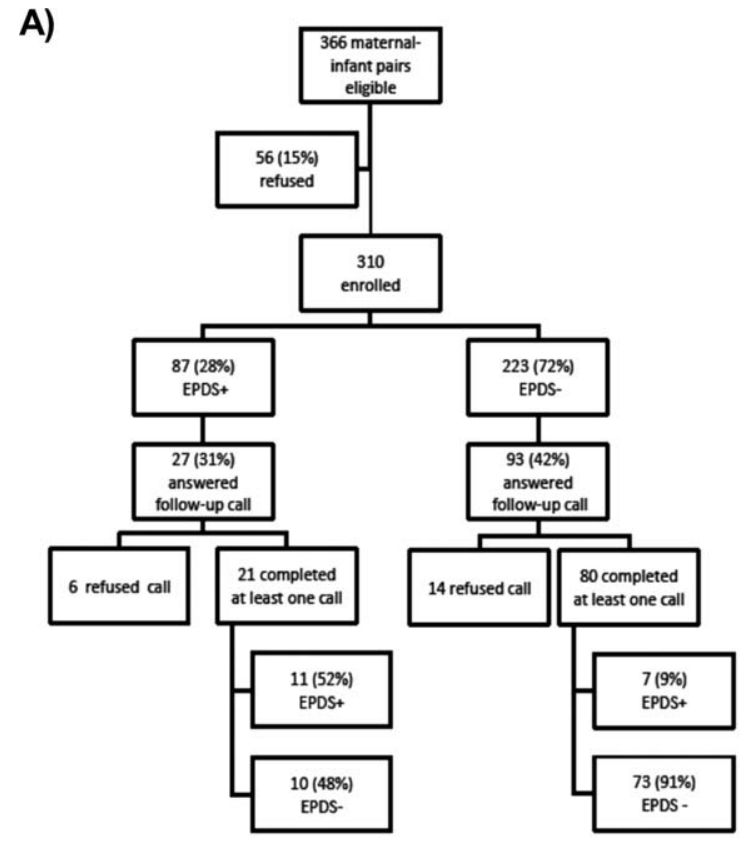

\section{B)}

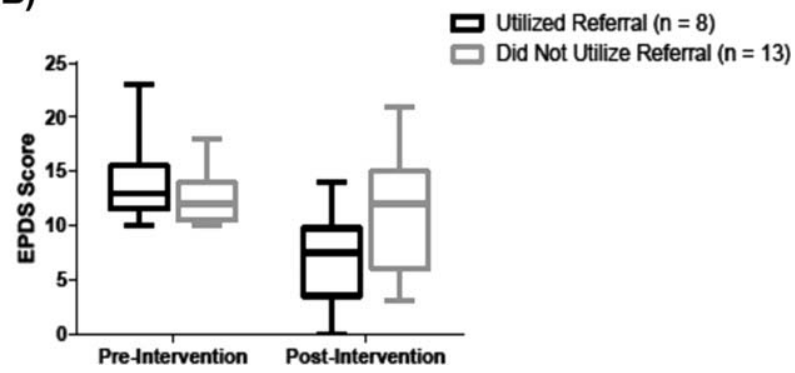

FIG. 1. (A) Flow diagram showing percentages of mothers completing each step of the study.(B) Postenrollment change in mean Edinburgh Postpartum Depression Scale (EPDS) score of all initially EPDS + mothers who completed at least 1 follow-up phone call, separated by if they did or did not seek referral. Mothers using referral (either spoke with physician or used resource sheet) had significantly larger reduction in score. Statistical analysis by analysis of variance, $P<0.05$.

women who did and did not seek further postpartum depression evaluation were compared via 2-way repeated measures analysis of variance. Statistical analyses were performed using R software. ${ }^{28}$

\section{RESULTS}

Out of 366 mother-infant pairs, $56(15 \%)$ refused, and $310(85 \%)$ mothers were fully enrolled (Figure 1A). Mothers had an average age of 28.17 years, were $68.3 \%$ Hispanic/Latina by self-report, and $45.2 \%$ were married. Infants were an average of 4.24 months old, $81.9 \%$ were born term (>37 weeks), and $64.8 \%$ were previously healthy (Table 1$)$.

Eighty-seven (28\%) mothers were EPDS+; 223 $(72 \%)$ were EPDS-. Only 42 mothers reported previous postpartum depression screening since the birth of their most recent child. However, 30 infants were $<1$ month in age, thus outside recommended screening range. Eliminating these infants revealed a $14.6 \%$ rate
TABLE 1. Maternal and Infant Characteristics

\begin{tabular}{lc}
\hline Characteristic & All Participants, $\mathrm{n}=310$ \\
\hline Maternal characterisitics & \\
Age, $\mathrm{y}^{*}$ & $28.17 \pm 6.18$ \\
Racelethnicicty & \\
White & $48(15.5 \%)$ \\
Black & $25(8.1 \%)$ \\
Hispanic & $211(68.3 \%)$ \\
Other & $25(8.1 \%)$ \\
EPDS language & \\
English & $231(74.5 \%)$ \\
Spanish & $79(25.5 \%)$ \\
People in homet & $5(4,6)$ \\
No. of childrent & $2(1,3)$ \\
Relationship & \\
Married & $140(45.2 \%)$ \\
In a relationship & $105(33.9 \%)$ \\
Single & $62(20 \%)$ \\
Any breastreeding & $142(45.8 \%)$ \\
Unsupportive social network & $54(17.4 \%)$ \\
Some psychiatric disorder & $47(15.2 \%)$ \\
MiB scoret & $6(3,10)$ \\
Infant characteristics & \\
Age, mo* & \\
Gestational age, wkt & $4.24 \pm 3.19$ \\
Prior admission & $39(37,40)$ \\
Any comorbidity & $113(36.5 \%)$ \\
Congenital heart disease & $109(35.2 \%)$ \\
Neurodeveloomental & $27(8.7 \%)$ \\
Any medical device needed & $22(7.1 \%)$ \\
& $38(12.3 \%)$
\end{tabular}

NOTE: Abbreviations: EPDS, Edinburgh Postpartum Depression Scale; MIB, maternal-infant bonding scale. ${ }^{*}$ The variable is summarized as mean \pm standard deviation. $†$ The variable is summarized as median (25th percentile, 75 th percentile)

of appropriate prior screening. Higher EPDS scores were associated with higher (worse) MIB scores by linear regression $(\beta=0.11, P<0.001)$. The vast majority $(77 \%)$ of mothers scored a 0 or 1 on the MIB scale, indicating good bonding; further statistical comparison using the MIB scale as a secondary outcome was therefore inappropriate.

On bivariate logistic regression, Hispanic/Latina women were less likely to be EPDS+ (odds ratio [OR]: 0.43 ; $95 \%$ CI: $0.23-0.84)$ compared to white/ Caucasian women. Mothers who identified Spanish as their primary language and took the Spanish EPDS had lower odds of a positive screen (OR: 0.47; $95 \%$ CI: $0.25-0.88)$. The racial differences did not persist on multivariate analysis (OR: 0.64 ; $95 \%$ CI: $0.30-$ 1.38) (Table 2). Maternal characteristics identified as potential risk factors for positive screens were poor social support (OR: 3.58; 95\% CI: 1.95-6.59) and history of a prior psychiatric diagnosis (OR: 5.07; 95\% CI: 2.65-9.72). There were no differences in age, number of children or people living in the home, relationship status, or breastfeeding rates by EPDS score.

Infant characteristics were next examined. Children of EPDS+ and EPDS - mothers were similar in age, number of prior hospital admissions, gestational age at birth, and overall use of medical equipment (Table 2). To examine the effect of illness leading to 
hospitalization on EPDS+ risk, discharge diagnoses were collected and grouped into categories. Infants of EPDS + mothers were more likely hospitalized for neurologic illness $(P=0.008)$ (see Supporting Table 1 in the online version of this article), but otherwise similar.

We next compared differences in long-term infant comorbidities. The rate of having any comorbidity was similar between children of EPDS+ and EPDS-

\begin{tabular}{|c|c|c|c|}
\hline & OR & $95 \% \mathrm{Cl}$ & $P$ Value \\
\hline \multicolumn{4}{|l|}{ Maternal characteristics } \\
\hline Maternal age & 0.99 & $0.95-1.03$ & 0.660 \\
\hline \multicolumn{4}{|l|}{ Race } \\
\hline White & Reference & - & - \\
\hline Black & 0.93 & $0.35-2.50$ & 0.891 \\
\hline Hispanic & 0.43 & $0.23-0.84$ & 0.013 \\
\hline Other & 0.54 & $0.19-1.55$ & 0.254 \\
\hline EPDS language & 0.47 & $0.25-0.88$ & 0.020 \\
\hline People in home & 1.02 & $0.89-1.16$ & 0.799 \\
\hline No. of children & 1.02 & $0.85-1.23$ & 0.819 \\
\hline \multicolumn{4}{|l|}{ Relationship } \\
\hline Married & Reference & - & - \\
\hline In a relationship & 0.93 & $0.52-1.65$ & 0.802 \\
\hline Single & 1.37 & $0.72-2.62$ & 0.333 \\
\hline Unsupportive social network & 3.58 & $1.95-6.59$ & $<0.0001$ \\
\hline Some psychiatric disorder & 5.07 & $2.65-9.72$ & $<0.0001$ \\
\hline \multicolumn{4}{|l|}{ Infant characteristics } \\
\hline Gestational age & 0.96 & $0.87-1.04$ & 0.316 \\
\hline Prior admission & 0.83 & $0.49-1.39$ & 0.476 \\
\hline Any comorbidity & 1.03 & $0.92-1.18$ & 0.551 \\
\hline Congenital heart disease & 1.87 & $0.83-4.22$ & 0.130 \\
\hline Neurodevelopmental & 3.41 & $1.41-8.21$ & 0.006 \\
\hline Any medical device needed & 1.59 & $0.78-3.24$ & 0.201 \\
\hline \multicolumn{4}{|l|}{ Multivariate logistic regression } \\
\hline \multicolumn{4}{|l|}{ Race } \\
\hline White & Reference & - & - \\
\hline Black & 0.87 & $0.28-2.70$ & 0.812 \\
\hline Hispanic & 0.64 & $0.30-1.38$ & 0.258 \\
\hline Other & 0.88 & $0.29-2.74$ & 0.831 \\
\hline Unsupportive social network & 4.40 & $2.27-8.53$ & $<0.0001$ \\
\hline Psychiatric disorder & 5.02 & $2.49-10.15$ & $<0.0001$ \\
\hline Neurodevelopmental comorbidity & 2.78 & $1.03-7.52$ & 0.004 \\
\hline
\end{tabular}

NOTE: Abbreviations: $\mathrm{Cl}$, confidence interval; EPDS, Edinburgh Postpartum Depression Scale; OR, odds ratio. mothers $(39.1 \%$ vs $33.6 \% ; P=0.551)$. However, children of EPDS + mothers were more likely to have mental retardation, hydrocephalus, or require ventriculoperitoneal shunt (VPS); however, the overall number of infants with each comorbidity was low. A neurodevelopmental comorbidity variable was created combining mental retardation, cerebral palsy, epilepsy, hydrocephalus, craniosynostosis, and VPS, resulting in $22(7.1 \%)$ unique infants with 1 or more of these conditions. Having an infant with a neurodevelopmental comorbidity was a risk factor for positive postpartum depression screen (OR: 3.41; 95\% CI: 1.41-8.21). This continued to be significant (OR: 2.78; 95\% CI: 1.03-7.52) (Table 2) when controlling for maternal race/ethnicity, psychiatric history, and social support in multivariate logistic regression.

To determine if women screened followed through with recommendations, participants were called 3 and 6 months postenrollment. We attempted to call all women and successfully reached 120; 19 (16\%) refused the call. One hundred one of the original 310 enrolled $(33 \%)$ completed at least 1 follow-up call; 47 at 3 months, 40 at 6 months, and only 14 (14\%) responded at both time points. Due to this response rate, the first call at either 3 or 6 months was used as a single follow-up time point for statistical analysis. A slightly higher proportion of EPDS-- mothers (80/ $223,36 \%$ ) completed calls compared to EPDS+ mothers $(21 / 87,24 \% ; P=0.047)$.

Of 21 mothers initially EPDS + who completed a follow-up call, $10(48 \%)$ later screened negative. Seven of these $10(70 \%)$ reported discussing postpartum depression with their physician or using provided referral resources in the interim; 1 woman both spoke to a doctor and used a referral resource. One additional woman used resources, but repeat EPDS was still positive (Table 3). Reasons cited for not seeking evaluation included "too busy" $(\mathrm{n}=4)$ and "lost paperwork" $(\mathrm{n}=1)$, or no reason was given $(\mathrm{n}=2)$. Mothers utilizing appropriate follow-up had reduction in scores compared to those not $(F(1,19)=5.743, P$ $=0.027)$, although all scores decreased over time $(F(1,19)=11.54, P=0.0030)($ Figure $1 \mathrm{~B})$.

\begin{tabular}{|c|c|c|c|}
\hline Changes in Characteristics Following Enrollment & Positive EPDS, $N=21$ & Negative EPDS, $\mathrm{N}=80$ & $P$ Value \\
\hline Repeat EPDS negative & $10(47.6 \%)$ & $73(91.3 \%)$ & $<0.001$ \\
\hline Spoke to a doctor about PD & $6(28.6 \%)$ & $27(33.7 \%)$ & 0.360 \\
\hline Used a study referral resource & $3(14.3 \%)$ & NA & \\
\hline Received a formal diagnosis of PD & $1(4.7 \%)$ & $1(1.3 \%)$ & 0.325 \\
\hline \multicolumn{4}{|l|}{ Healthcare utilization* } \\
\hline NNo. of ER visits & $0(0-0.5)$ & $0(0-2)$ & 0.074 \\
\hline №. of urgent care visits & $0(0-0.5)$ & $0(0-0)$ & 0.136 \\
\hline No. of hospitalizations & $0(0-0)$ & $0(0-1)$ & 0.021 \\
\hline Repeat MIB score & $1.09 \pm 0.38$ & $0.69 \pm 0.17$ & 0.357 \\
\hline
\end{tabular}

NOTE: Abbreviations: EPDS, Edinburgh Postpartum Depression Scale; ER, emergency room; MIB, maternal-infant bonding scale; NA, not applicable; PD, postpartum depression. *The variable is summarized as median (interquartile range). 
Of 80 women initially EPDS-, most stayed negative $(73 / 80,91 \%)$, but 7 (9\%) became EPDS + . These mothers received education and referral information over the phone, but none completed a subsequent call. Infants of mothers initially EPDS - had a higher frequency of hospitalization postenrollment compared to EPDS + mothers $(P=0.021)$ (Table 3$)$. Two $(33 \%)$ mothers who converted from EPDS - to EPDS + had infants readmitted in the follow-up period.

\section{DISCUSSION}

This study demonstrated almost a third of mothers of hospitalized infants are at risk for postpartum depression and most had not been previously screened. Stress due to hospitalization did not seem to falsely elevate EPDS scores; the proportion of EPDS + mothers matched our prestudy prediction ( $28 \%$ vs $27.9 \%$ ). Follow-up calls indicated that EPDS + mothers not pursuing further evaluation tended to remain EPDS+. Higher (worse) MIB score was strongly correlated to increased EPDS score as expected, supporting screening accuracy. Our results suggest that postpartum depression screening in hospital settings can be used to complement outpatient practice and capture mothers who would otherwise be missed.

Although we were able to screen, it is difficult to know whether this correctly identified mothers with postpartum depression. Only 2 mothers reported subsequent official diagnosis of postpartum depression, and 1 of these was EPDS - originally. This reflects weakness of our survey-based design; we only know if the mother self-reported a formal diagnosis of postpartum depression, because we do not have access to their medical charts. We also had higher than expected loss to follow up (67\%), leaving 66 initially EPDS + mothers with unknown eventual diagnoses. The EPDS has been validated in multiple populations and has a positive predictive value ranging from $23 \%$ to $93 \% .{ }^{23}$ Therefore, somewhere between 20 and 80 women in our study should meet diagnostic criteria for postpartum depression. A limitation of children's hospital-based screening with the EPDS is lack of adult-trained psychiatrists who could immediately follow screening with diagnosis. Such integration may already be possible at community or hospital-withina-hospital models, and could be trialed at children's hospitals. Regardless, participation in the study seemed to increase mothers' awareness of postpartum depression. Prior to enrollment, only $14.6 \%$ of subjects reported discussing postpartum depression with a physician, although recall bias likely contributed to some mothers not remembering a screen. Promisingly, on follow-up, $37 \%$ of called participants reported they discussed postpartum depression with a doctor following their child's hospital discharge.

Our study identified low social support and history of past psychiatric diagnosis as maternal risk factors for EPDS+ screens, which is consistent with previous reports. ${ }^{29}$ There was a slight increase in subsequent infant hospitalizations in the EPDS - group, which is contrary to reports stating that increased healthcare utilization is associated with postpartum depression. ${ }^{30}$ However, most studies have shown an increase in only acute or emergency room care visits ${ }^{30,31}$ and no association between maternal depression and infant hospitalization. ${ }^{30,32}$ In our study, the median number of hospitalizations for both groups was 0 , indicating overall low utilization. Because 2 of the mothers who converted from EPDS - to EPDS + had children readmitted, this underscores the benefit of reassessment at each medical encounter. A large proportion of mothers $(36.5 \%)$ reported that the infant had been previously hospitalized, adding another potential missed screening opportunity. Our study supports others advocating repeated screenings and suggests mothers should be screened at any medical encounter that occurs in the first postpartum year.

We identified neurodevelopmental illness as the major infant characteristic associated with postpartum depression risk. Conversely, Garfield et al. did not find correlation between poorer Neurobiologic Risk Score and increased maternal depression risk in a NICU setting. ${ }^{11}$ Perhaps our population of older and mainly full-term infants makes consequences of neurologic insult more obvious and affects mothers more significantly. Cheng et al. reported that $26.9 \%$ of mothers of children with cognitive delay reported high depressive symptoms, compared with $17.4 \%$ of mothers of typically developing children at 4 years of age. ${ }^{33}$ Another body of evidence suggests maternal emotional state during pregnancy influences neurodevelopmental outcome in the child. Maternal anxiety or depression has been associated with altered placental function, reduced infant gray matter density, and worse cognitive function. ${ }^{34,35}$ Therefore, future research may focus on mothers of infants with neurodevelopmental disease to better understand this relationship.

There were several limitations to this study. Some data collected by a survey are subject to information bias. Women may report a more supportive social network than actually exists or omit history of mental health diagnoses. We attempted to control for this by using validated measures where possible and performing chart review to verify reported infant characteristics. Our population was overwhelmingly Hispanicl Latina, and a third of infants were not previously healthy, which limits applicability to other settings. We used a convenience method that could introduce sampling bias. Our hospital's overall patient demographic is $65 \%$ Hispanic, which is similar to the $68 \%$ sampled in our study. In addition, the proportions of infant diagnoses approximate the overall rates at CHLA, so we feel our sample was fairly representative. There is a general consensus that depression studies have recruitment difficulties. ${ }^{36}$ In the unlikely event that all 56 of women who declined to 
participate were EPDS+, overall proportion of at-risk mothers would rise to $39 \%$. If our study does show slight underestimation of risk, that would only mean more potential for intervention if screening were mandatory. Another weakness was high loss to follow-up, which led us to combine the 3- and 6-month followup calls into 1 outcome. Sixty percent of calls used in analysis occurred at 3 months, so long-term maintenance of improved EPDS scores remains unclear. Although conducting repeat EPDS via phone may affect honest answering of sensitive questions, other studies have used this technique successfully. ${ }^{4}$

\section{CONCLUSION}

This is the first study evaluating a screening program for maternal postpartum depression during infant hospitalizations. In our population, risk factors for positive postpartum depression screening were low social support, history of maternal psychiatric diagnosis, and having an infant with neurodevelopmental disease. We believe mothers should receive postpartum depression screening at all medical encounters during the child's first year.

\section{Acknowledgements}

The authors thank the CHLA Department of Social Work and the USC Required Scholarly Projects program, and specifically Joseph DeSena and Humberto Avila, for project assistance.

Disclosures: Dr. Trost is an Institutional Career Development Program Scholar through the Southern California Clinical and Translational Science Institute (SC-CTSI) at the University of Southern California Keck School of Medicine. The content is solely the responsibility of the author(s) and does not represent the official view of the SC-CTSI. Dr. Trost conceptualized and designed the study, drafted the initial manuscript, and approved the final manuscript as submitted. Dr. MolasTorreblanca co-designed the study, reviewed and revised the manuscript, and approved the final manuscript as submitted. Ms. Man coordinated and supervised hospital data collection, critically reviewed the manuscript, and approved the final manuscript as submitted. Mr. Casillas coordinated and supervised the phone call data collection, critically reviewed the manuscript, and approved the final manuscript as submitted. Ms. Sapir coordinated the referral process for enrolled patients, supervised the design of patient handouts, and critically reviewed and approved the final manuscript as submitted. Dr. Schrager guided study design, supervised the statistical analysis of the final data, critically reviewed and revised the manuscript, and approved the final manuscript as submitted. The authors report no conflicts of interest.

\section{References}

1. Chung EK, McCollum KF, Elo IT, Lee HJ, Culhane JF. Maternal depressive symptoms and infant health practices among low-income women. Pediatrics. 2004;113(6):e523-e529.

2. Myers ER, Aubuchon-Endsley N, Bastian LA, et al.; Duke University Evidence-based Practice Center. Effective Health Care Program. Efficacy and safety of screening for postpartum depression. Comparative effectiveness review number 106. Rockville, MD: Agency for Healthcare Research and Quality, U.S. Department of Health and Human Services; 2013: Available at: https://www.effectivehealthcare.ahrq. gov/ehc/products/379/1437/postpartum-screening-report-130409.pdf. Date accessed Jan 102016.

3. Gotlib IH, Whiffen VE, Mount JH, Milne K, Cordy NI. Prevalence rates and demographic characteristics associated with depression in pregnancy and the postpartum. J Consult Clin Psychol. 1989;57(2): 269-274.

4. Hanusa BH, Scholle SH, Haskett RF, Spadaro K, Wisner KL. Screening for depression in the postpartum period: a comparison of three instruments. J Womens Health (Larchmt). 2008;17(4):585-596.

5. Bogen DL, Hanusa BH, Moses-Kolko E, Wisner KL. Are maternal depression or symptom severity associated with breastfeeding intention or outcomes? J Clin Psychiatry. 2010;71(8):10691078.
6. Surkan PJ, Ettinger AK, Ahmed S, Minkovitz CS, Strobino D. Impact of maternal depressive symptoms on growth of preschool- and schoolaged children. Pediatrics. 2012;130(4):e847-e855.

7. McLearn KT, Minkovitz CS, Strobino DM, Marks E, Hou W. The timing of maternal depressive symptoms and mothers' parenting practices with young children: implications for pediatric practice. Pediatrics. 2006;118(1):e174-e182.

8. Shaw DS, Connell A, Dishion TJ, Wilson MN, Gardner F. Improvements in maternal depression as a mediator of intervention effects on early childhood problem behavior. Dev Psychopathol. 2009;21(2): 417-439.

9. Earls MF; Committee on Psychosocial Aspects of Child and Family Health American Academy of Pediatrics. Incorporating recognition and management of perinatal and postpartum depression into pediatric practice. Pediatrics. 2010;126(5):1032-1039.

10. Birmingham MC, Chou KJ, Crain EF. Screening for postpartum depression in a pediatric emergency department. Pediatr Emerg Care. 2011;27(9):795-800.

11. Garfield L, Holditch-Davis D, Carter CS, et al. Risk factors for postpartum depressive symptoms in low-income women with very lowbirth-weight infants. Adv Neonatal Care. 2015;15(1):E3-E8.

12. Ueda M, Yamashita H, Yoshida K. Impact of infant health problems on postnatal depression: pilot study to evaluate a health visiting system. Psychiatry Clin Neurosci. 2006;60(2):182-189.

13. Olson AL, Kemper KJ, Kelleher KJ, Hammond CS, Zuckerman BS, Dietrich AJ. Primary care pediatricians' roles and perceived responsibilities in the identification and management of maternal depression. Pediatrics. 2002;110(6):1169-1176.

14. Head JG, Storfer-Isser A, O'Connor KG, et al. Does education influence pediatricians' perceptions of physician-specific barriers for maternal depression? Clin Pediatr (Phila). 2008;47(7):670-678.

15. Wiley CC, Burke GS, Gill PA, Law NE. Pediatricians' views of postpartum depression: a self-administered survey. Arch Womens Ment Health. 2004;7(4):231-236.

16. Selden TM. Compliance with well-child visit recommendations: evidence from the Medical Expenditure Panel Survey, 2000-2002. Pediatrics. 2006;118(6):e1766-e1778.

17. Berry JG, Blaine K, Rogers J, et al. A framework of pediatric hospital discharge care informed by legislation, research, and practice. JAMA Pediatr. 2014;168(10):955-962; quiz 965-966.

18. Fisher ES, Rosenbluth G, Shaikh U. Pedi-BOOST. Peds Effective Discharge: Better Handoff to Home through Safer Transitions. 2013. https:// www.hospitalmedicine.org/Web/Quality__Innovation/Implementation Toolkit/pediBoost/Best_Practices/Best_Practices.aspx Accessed Jan 10 2016.

19. Ohoka H, Koide T, Goto S, et al. Effects of maternal depressive symptomatology during pregnancy and the postpartum period on infant-mother attachment. Psychiatry Clin Neurosci. 2014;68(8):631639.

20. Duggan AK, Berlin LJ, Cassidy J, Burrell L, Tandon SD. Examining maternal depression and attachment insecurity as moderators of the impacts of home visiting for at-risk mothers and infants. J Consult Clin Psychol. 2009;77(4):788-799.

21. Nonacs R, Cohen LS. Postpartum mood disorders: diagnosis and treatment guidelines. J Clin Psychiatry. 1998;59(suppl 2):34-40.

22. Taylor A, Atkins R, Kumar R, Adams D, Glover V. A new Mother-toInfant Bonding Scale: links with early maternal mood. Arch Womens Ment Health. 2005;8(1):45-51.

23. Gibson J, McKenzie-McHarg K, Shakespeare J, Price J, Gray R. A systematic review of studies validating the Edinburgh Postnatal Depression Scale in antepartum and postpartum women. Acta Psychiatr Scand. 2009;119(5):350-364.

24. Cox JL, Holden JM, Sagovsky R. Detection of postnatal depression. Development of the 10-item Edinburgh Postnatal Depression Scale. $\mathrm{Br}$ J Psychiatry. 1987;150:782-786.

25. Garcia-Esteve L, Ascaso C, Ojuel J, Navarro P. Validation of the Edinburgh Postnatal Depression Scale (EPDS) in Spanish mothers. J Affect Disord. 2003;75(1):71-76.

26. Yawn BP, Dietrich AJ, Wollan P, et al. TRIPPD: a practice-based network effectiveness study of postpartum depression screening and management. Ann Fam Med. 2012;10(4):320-329.

27. Chaudron LH, Szilagyi PG, Kitzman HJ, Wadkins HI, Conwell Y. Detection of postpartum depressive symptoms by screening at wellchild visits. Pediatrics. 2004;113(3 pt 1):551-558.

28. R Core Team. R: A language and environment for statistical computing. Vienna, Austria: R Foundation for Statistical Computing: 2013. Available at: http://www.R-project.org. Accessed Jan 10 2016.

29. Yim IS, Tanner Stapleton LR, Guardino CM, Hahn-Holbrook J, Dunkel Schetter C. Biological and psychosocial predictors of postpartum depression: systematic review and call for integration. Annu Rev Clin Psychol. 2015;11:99-137.

30. Minkovitz CS, Strobino D, Scharfstein D, et al. Maternal depressive symptoms and children's receipt of health care in the first 3 years of life. Pediatrics. 2005;115(2):306-314. 
31. Watson JM, Kemper KJ. Maternal factors and child's health care use. Soc Sci Med. 1995;40(5):623-628.

32. Kahn RS, Zuckerman B, Bauchner H, Homer CJ, Wise PH. Women's health after pregnancy and child outcomes at age 3 years: a prospective cohort study. Am J Public Health. 2002;92(8):1312-1318.

33. Cheng ER, Palta M, Poehlmann-Tynan J, Witt WP. The influence of children's cognitive delay and behavior problems on maternal depression. J Pediatr. 2015;167(3):679-686.

34. Blakeley PM, Capron LE, Jensen AB, O’Donnell KJ, Glover V. Maternal prenatal symptoms of depression and down regulation of placental monoamine oxidase A expression. J Psychosom Res. 2013;75(4):341345 .

35. Buss C, Davis EP, Muftuler LT, Head K, Sandman CA. High pregnancy anxiety during mid-gestation is associated with decreased gray matter density in 6-9-year-old children. Psychoneuroendocrinology. 2010;35(1):141-153.

36. Hughes-Morley A, Young B, Waheed W, Small N, Bower P. Factors affecting recruitment into depression trials: Systematic review, metasynthesis and conceptual framework. J Affect Disord. 2015;172:274290. 